artery thrombosis. These exclusions mean that the results are not strictly intention to treat, but the number of patients excluded is small (11 in total) and therefore unlikely to bias the results.

As with all meta-analyses of published data, there is the potential problem of publication bias. We did a comprehensive literature search to identify published and unpublished data. It therefore seems unlikely that this would be a substantial source of bias.

Contributors: See bmj.com

Funding: No funding was sought for this study.

Competing interests: DA is an investigator in a trial of fluvastatin in renal transplant recipients that is funded by Novartis; PC and DA have a grant from Roche to study chronic allograft nephropathy; PC has an unrestricted educational grant from Novartis to support research into early immune events after renal transplantation, has received support from Roche and Novartis to attend transplant meetings, and has spoken at educational meetings sponsored by these companies.

UK Transplant website. www.uktransplant.org (accessed 25 February 2003).

2 Hariharan S, Johnson C, Bresnahan B, Taranto S, McIntosh M, Stablein D. Improved graft survival after renal transplantation in the United States, 1988 to 1996. N Engl J Med 2000;342:605-12.

3 Matas A, Gillingham K, Payne W, Najarian J. The impact of an acute rejection episode on long-term renal allograft survival. Transplantation 1994;57:857-9.

4 Kamel O, van de Rijn M, Lebrun D, Weiss L, Warnke R, Dorfman R. A multivariate analysis of discharge immunosuppression and posttransplant malignancy. Am J Transplant 2001;1(suppl 1):353

5 Jamil B, Nicholls K, Becker G, Walker R. Impact of acute rejection therapy on infections and malignancies in renal transplant recipients. Transplantation 1999;68:1597-619.

\section{What is already known on this topic}

Episodes of acute rejection reduce graft survival in patients with renal transplants

Increasing immunosuppression to reduce rejection can increase infection and malignancy

\section{What this study adds}

Addition of interleukin-2 receptor antibodies to ciclosporin based immunosuppression regimens halves the risk of acute rejection

Patients receiving antibodies did not have an increased risk of infection

The effects on graft loss and mortality at one year were not significant

6 Morgan D, Ruscettis F, Gallo R. Selective in vitro growth of T lymphocytes from normal human bone marrow. Science 1976;193:1007-8.

7 Robb R, Greene W, Rusk C. Low and high affinity cellular receptors for interleukin 2: implications for the level of Tac antigen. J Exp Med $1984 ; 162: 358-62$.

8 Kirkman R, Barrett L, Carpenter C, McKay DB, Milford EL, Ramos EL, et al. A randomised trial of anti-Tac monoclonal antibody in human renal transplantation. Transplantation 1991;51:107-13.

9 Van Gelder T, Zietse R, Mulder A, Yzermans JNM, Hesse CJ, Vaessen LMB, et al. A double blind, placebo-controlled study of monoclonal antiinterleukin-2 receptor antibody (BT563) administration to prevent acute rejection after kidney transplantation. Transplantation 1995;60:248-52.

10 Knoll G, Bell R. Tacrolimus versus cyclosporin for immunosuppression in renal transplantation: meta-analysis of randomised trials. BMJ 1999:318:1104-7.

(Accepted 14 February 2003)

\title{
RESEARCH POINTERS
}

\section{Risk of subsequent thromboembolism for patients with pre-eclampsia}

\author{
Carl van Walraven, Muhammad Mamdani, Adam Cohn, Yasir Katib, Mark Walker, Marc A Rodger
}

Inherited thrombophilias affect over $16 \%$ of people and predispose them to venous thromboembolism. ${ }^{1}$ Pre-eclampsia is associated with occlusion of the placental spiral arteries. ${ }^{2}$ Thrombophilias may cause thrombosis of placental vessels, ${ }^{3}$ thereby explaining the link between thrombophilia and pre-eclampsia. ${ }^{45}$ We tested the hypothesis that women with pre-eclampsia have a higher risk of subsequent venous thromboembolism.

\section{Participants, methods, and results}

The study was approved by our research ethics board and took place in Ontario, Canada, where all hospital services are publicly funded. We used an administrative database that is based on anonymised populations and which records all hospitalisations from 1 April 1988 onwards. All discharges between 1 April 1990 and 1 January 1994 with a primary ICD-9 (international classification of diseases, 9th revision) code of pre-eclampsia, severe pre-eclampsia, eclampsia, or toxaemia were identified (see appendix A on bmj.com). These codes had a sensitivity of $89 \%$
(95\% confidence interval $78 \%$ to $94 \%$ ) and a specificity of $67 \%$ ( $79 \%$ to $94 \%$ ) for patients with true pre-eclampsia (appendix B).

We used multiple control groups consisting of women with the 10 most common primary obstetrical diagnoses at time of discharge (appendix A; see figure). We determined from the hospitalisation database whether patients had had a caesarean section during their index hospitalisation. If they had had one or more admissions for pre-eclampsia, they were assigned to the pre-eclampsia group. Patients were excluded if they were less than 15 years old or if they were admitted to hospital for deep vein thrombosis or pulmonary embolism (appendix A) before entering the study. These admissions were also identified in the hospitalisations database.

The observation period started when patients were discharged from their index hospitalisation and ended at study end (1 January 1995), when patients died, or when patients were admitted with venous thromboembolism. We chose 1995 as study end because until 1995 patients with thromboembolism were initially treated in hospital with unfractionated
Thrombosis of placental vessels causes pre-eclampsia. Women with pre-eclampsia had an increased risk of subsequent venous thromboembolism

continued over BMJ 2003;326:791-2

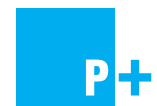

More information on ICD-9 codes can be found on bmj.com 
Ottawa Health

Research Institute,

Ottawa, Canada

K1Y 4E9

Carl van Walraven

scientist

Marc A Rodger

associate scientist

Mark Walke

associate scientist

Institute for Clinical

Evaluative Sciences

Toronto, Canada

M4N 3N5

Muhammad

Mamdani

scientist

University of

Ottawa, Ottawa,

Canada K1H 8M5

Adam Cohn

resident

Yasir Katib

resident

Correspondence to:

C van Walraven

carlv@ohri.ca

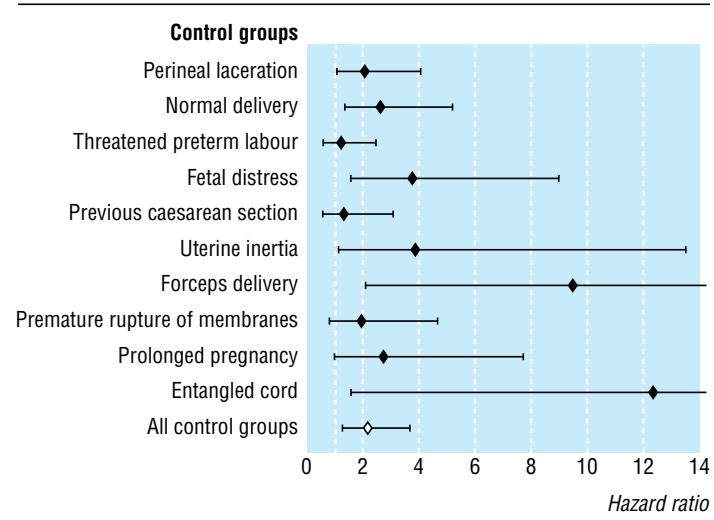

Independent risk of venous thromboembolism for women with pre-eclampsia. The adjusted hazard ratio for admission to hospital with deep vein thrombosis or pulmonary embolism is presented for women with pre-eclampsia compared with the 10 most common obstetrical discharge diagnoses. The hazard ratios (shown here with

95\% confidence intervals) are adjusted for patient age and whether a caesarean section was performed at the index admission

heparin. This ensures that virtually all such patients were admitted to hospital and identified in the hospitalisation database (appendix C). Dates of death were determined from the registered patient database that records all deaths in Ontario.

During the study period, 297912 women were discharged from hospital with one of the study diagnoses. Some were excluded because they were under 15 or had previous thromboembolic disease ( $\mathrm{n}=703$ and $\mathrm{n}=172$, respectively). This left 12849 women in the pre-eclampsia group and 284188 women in the control groups (range 11188 to 22 883). Patients with pre-eclampsia were younger (mean $27.8 v 28.1$ ) and more likely to have had a caesarean section $(20.0 \% v 10.6 \%)$. Mean follow up was three years.

Venous thromboembolism was more common in the pre-eclampsia group $(0.12 \%, 41.7$ events per 100000 person years observation) than in any of the control groups (range $0.01 \%$ to $0.08 \%$, rates of 3.0 to 33.8 events per 100000 person years observation). We used proportional hazards modelling to control for patient age and caesarean section, and showed that, compared with all control groups combined, women with pre-eclampsia were 2.2 times more likely (95\% confidence interval 1.3 to 3.7 ) to be admitted to hospital with venous thromboembolism (figure). The proportional hazards assumption was valid for all models.

\section{Comment}

Women with pre-eclampsia have a small but significantly higher risk of subsequent venous thromboembolic disease compared with women diagnosed as having other common obstetrical diseases. Our results support the association between pre-eclampsia and thrombophilia.

The absolute risk increase with pre-eclampsia is too small to warrant venous thromboembolism prophylaxis for such patients. The signs and symptoms of venous thromboembolism should be reviewed with women who develop or have had pre-eclampsia so that they can seek appropriate medical care if the need arises.

Contributors: CvW coordinated the study, drafted the paper, and was primarily responsible for data linkage and analysis. MM critically revised the paper, and assisted in data linkage and analysis. AC coordinated the reabstraction study and reviewed the paper. YK coordinated the reabstraction study. MW and MR devised the study, provided expert content, and critically revised the paper. $\mathrm{CvW}$ is the guarantor.

Funding: CvW and MW are Ontario Ministry of Health career scientists

Competing interests: None declared.

1 Rodeghiero F, Tosetto A. The epidemiology of inherited thrombophilia: the VITA Project. Vicenza Thrombophilia and Atherosclerosis Project. Thromb Haemost 1997;78:636-40.

2 Redman CW. Platelets and the beginnings of preeclampsia. N Engl J Med 1990;323:478-80.

3 Gris JC, Ripart-Neveu S, Maugard C, Tailland ML, Brun S, Courtieu C, et al. Respective evaluation of the prevalence of haemostasis abnormalities in unexplained primary early recurrent miscarriages. The Nimes obstetricians and haematologists (NOHA) study. Thromb Haemost 1997;77:1096-103

4 Grandone F, Margaglione M, Colaizzo D, Cappucci G, Paladini D, Martinelli P, et al. Factor V Leiden, C > T MTHFR polymorphism and genetic susceptibility to preeclampsia. Thromb Haemost 1997;77:1052-4.

5 Kupferminc MJ, Eldor A, Steinman N, Many A, Bar-Am A, Jaffa A, et al. Increased frequency of genetic thrombophilia in women with complications of pregnancy. N Engl J Med 1999;340:9-13.

(Accepted 1 August 2002)

\section{He died "peacefully" at home}

I like collecting euphemisms. I found a new one when my father, a retired general practitioner, died "peacefully" at home. That is what the announcements say in the newspapers, and that is what my relatives are telling everyone.

It was a perfectly routine death, in which my 93 year old father had cancer of the prostrate. The cancer had been well controlled for many years, and his health had started to deteriorate only in the few months before his death. Four days before his death, he was still able to go out to a celebration lunch. He then had a massive cerebrovascular accident, and there followed a predictable course of death by bronchopneumonia.

The last time I had experienced a death at such close quarters was many years ago as a houseman, but then I was not so personally involved. My family and father received excellent home care, with a hospital bed and visits by the district nurses and his general practitioner. Except that his death didn't seem to me to be "peaceful" at all. When my patients die they usually do so "violently," but at least it is sudden and they are spared a potentially drawn out course perhaps over many weeks.

We experienced a relatively short three days and nights of distressed breathing and bubbling in his lungs. Most of the time he was unable to communicate other than by raising his right hand and grasping any proffered hand. The final day was followed by seizures and increasing cyanosis, as he finally suffocated from his sputum. My mother wailed and implored him not to leave her. Leaving aside my mother's distress, surely there must be a better way of dying "peacefully"? Euphemisms always hide the painful truth, and this was one.

David Veale 